\title{
Ocular blood flow decreases during passive heat stress in resting humans
}

Tsukasa Ikemura ${ }^{1,2}$, Akane Miyaji $^{1,2}$, Hideaki Kashima' ${ }^{1}$, Yuji Yamaguchi ${ }^{1}$ and Naoyuki Hayashi ${ }^{2 *}$

\begin{abstract}
Background: Heat stress induces various physiological changes and so could influence ocular circulation. This study examined the effect of heat stress on ocular blood flow.

Findings: Ocular blood flow, end-tidal carbon dioxide $\left(P_{\mathrm{ET}} \mathrm{CO}_{2}\right)$ and blood pressure were measured for 12 healthy subjects wearing water-perfused tube-lined suits under two conditions of water circulation: (1) at $35^{\circ} \mathrm{C}$ (normothermia) for $30 \mathrm{~min}$ and (2) at $50^{\circ} \mathrm{C}$ for $90 \mathrm{~min}$ (passive heat stress). The blood-flow velocities in the superior temporal retinal arteriole (STRA), superior nasal retinal arteriole (SNRA), and the retinal and choroidal vessels (RCV) were measured using laser-speckle flowgraphy. Blood flow in the STRA and SNRA was calculated from the integral of a cross-sectional map of blood velocity. $P_{\mathrm{ET}} \mathrm{CO}_{2}$ was clamped at the normothermia level by adding $5 \% \mathrm{CO}_{2}$ to the inspired gas. Passive heat stress had no effect on the subjects' blood pressures. The blood-flow velocity in the RCV was significantly lower after 30,60 and 90 min of passive heat stress than the normothermic level, with a peak decrease of $18 \pm 3 \%$ (mean \pm SE) at $90 \mathrm{~min}$. Blood flow in the STRA and SNRA decreased significantly after 90 min of passive heat stress conditions, with peak decreases of $14 \pm 3 \%$ and $14 \pm 4 \%$, respectively.
\end{abstract}

Conclusion: The findings of this study suggest that passive heat stress decreases ocular blood flow irrespective of the blood pressure or arterial partial pressure of $\mathrm{CO}_{2}$.

Keywords: Retinal circulation, Choroidal circulation, Hyperthermia

\section{Background}

The ocular circulation consists of the choroidal and retinal vasculatures. Both blood vessels branch from the ophthalmic artery [1]. The ocular circulation nourishes the retina, which plays an important role in vision [2]. We have previously reported that changes in retinal and choroidal blood flow are associated with a change in visual acuity [3]. In addition, concomitant increases in choroidal blood flow and contrast sensitivity were found in healthy subjects after sildenafil administration [4]. Thus, changes in ocular blood flow can influence the visual function.

Heat stress elevates body temperature, which can lead to hyperventilation and a consequent reduction in the arterial partial pressure of carbon dioxide $\left(\mathrm{PaCO}_{2}\right)$ [5]. Since the retinal and choroidal blood vessels are sensitive to variations in $\mathrm{PaCO}_{2}$ [2], heat stress may cause a

\footnotetext{
* Correspondence: naohayashi@hum.titech.ac.jp

${ }^{2}$ Graduate School of Decision Science and Technology, Tokyo Institute of

Technology, Ookayama 2-12-1-W9-1, Meguro, Tokyo 152-8552, Japan

Full list of author information is available at the end of the article
}

reduction in flow in both ocular vessels. We previously reported that ocular blood flow in both vessels decreased concomitantly with hypocapnia during hyperventilation [3]; however, the effect of passive heat stress alone on ocular blood flow has yet to be examined.

It has been found that retinal blood flow decreased and choroidal blood flow was suppressed during exhaustive exercise both under hot and thermoneutral conditions [6]. This response was larger under the hot condition than in the thermoneutral condition, despite both conditions inducing a similar decrease in $\mathrm{PaCO}_{2}$, implying that additional passive heat stress can induce a further reduction in ocular blood flow. Thus, the purpose of the present study was to test the hypothesis that retinal and choroidal blood flow is decreased by passive heat stress for constant $\mathrm{PaCO}_{2}$. The flow response to passive heat stress in both of these ocular blood vessels was assessed under eucapnic conditions.
Ciomed Central

(C) 2013 Ikemura et al.; licensee BioMed Central Ltd. This is an Open Access article distributed under the terms of the Creative Commons Attribution License (http://creativecommons.org/licenses/by/2.0), which permits unrestricted use, distribution, and reproduction in any medium, provided the original work is properly cited. The Creative Commons Public Domain Dedication waiver (http://creativecommons.org/publicdomain/zero/1.0/) applies to the data made available in this article, unless otherwise stated. 


\section{Methods}

\section{Subjects}

The subjects were 12 healthy males (age, $24 \pm 2$ years (mean $\pm \mathrm{SD}$ ); height, $172 \pm 8 \mathrm{~cm}$; body mass $61 \pm 6 \mathrm{~kg}$ ). All of the subjects were free of any known autonomic dysfunction or cardiovascular or ocular disease, and were not taking any medication. The Ethics Committee of the Institution of Health Science, Kyushu University, Japan, approved the experimental protocol, and all subjects provided written informed consent to participate prior to the commencement of the study. All of the protocols used conformed to the Declaration of Helsinki.

\section{Protocol}

The subjects were asked to abstain from caffeinated beverages and strenuous exercise for $6 \mathrm{~h}$, and from eating for at least $2 \mathrm{~h}$ before the experiment. Following arrival at the laboratory, each subject put on a water-perfused tube-lined suit that covered the entire body except for the head, hands and feet. Initially, thermoneutral water $\left(35^{\circ} \mathrm{C}\right)$ was circulated through the tube-lined suit for 30 min (normothermia). The subjects were then exposed to passive whole-body heat by raising the temperature of the circulating water to $50^{\circ} \mathrm{C}$ for $90 \mathrm{~min}$. All subjects were required to maintain a resting state in a seated position throughout the experimental procedure.

Each subject's blood pressure, finger skin blood flow (fSBF) and heart rate (HR) were recorded continuously throughout the trial. The respiratory variables, ocular blood velocity and external ear temperature were recorded every 10 min during normothermia, and at 30 , 60 and $90 \mathrm{~min}$ after the induction of passive heat stress. In addition, to ensure that the end-tidal carbon dioxide $\left(P_{\mathrm{ET}} \mathrm{CO}_{2}\right)$ remained stable, the respiratory variables were measured at 20, 50 and $80 \mathrm{~min}$ after the induction of passive heat stress. When hypocapnia was observed, $5 \%$ $\mathrm{CO}_{2}$ gas was added to the inspired gas until the $P_{\mathrm{ET}} \mathrm{CO}_{2}$ returned to the normothermic level. The subjects were asked to open their eyes without blinking for $6 \mathrm{~s}$ during the recording of the image for the ocular blood flow measurement.

\section{Measurements}

The beat-by-beat blood pressure was monitored with an automatic sphygmomanometer attached to the left middle finger (Finometer, Finapres Medical Systems, Amsterdam, The Netherlands). An electrocardiogram was recorded continuously using a bioelectrical amplifier (MEG2100, Nihon-Kohden, Tokyo, Japan). The fSBF was measured using a laser Doppler flowmeter (FLO-CI, Omegawave, Tokyo, Japan). The temperature of the right external ear was measured using an infrared thermometer (Omron, Kyoto, Japan). The HR and mean arterial pressure (MAP) were calculated from the bioelectrical amplifier and blood-pressure recordings. The averaged data of the last minute in each of the measurement periods was used for analysis.

The subjects wore a nose clip and breathed through a mouthpiece that was connected to a hot-wire flowmeter (RM-300, Minato Medical Sciences, Osaka, Japan) to measure tidal volume $\left(V_{\mathrm{T}}\right)$ and $P_{\mathrm{ET}} \mathrm{CO}_{2}$. The flowmeter was calibrated using a 2-l syringe. Samples of respired gas $(1 \mathrm{ml} / \mathrm{s})$ were regularly drawn from the mouthpiece and analyzed for $\mathrm{CO}_{2}$ using a mass spectrometer (WSMR-1400, Westron, Chiba, Japan), which was calibrated with fresh air and precision gases. $\mathrm{PaCO}_{2}$ was estimated from $V_{\mathrm{T}}$ and $P_{\mathrm{ET}} \mathrm{CO}_{2}$ [7].

Laser-speckle images were obtained using a laserspeckle flowgraphy (LSFG) system (SoftCare, Fukuoka, Japan), which is described elsewhere (for example, [8]). The LSFG measurements were made in triplicate at each time point and the mean of the three values was used for analysis. Ocular blood-velocity data were obtained from the retinal and choroidal vasculature (RCV), the superior temporal retinal arteriole (STRA) and the superior nasal retinal arteriole (SNRA). The RCV accounts for most of the choroidal circulation, since the choroidal blood flow constitutes $85 \%$ of the total ocular blood flow to the retina [9]. The STRA and SNRA supply blood to the retina [1]. Blood flow in the STRA and SNRA was calculated from the integral of a cross-sectional map of blood velocity within the selected arteriole. The conductance index (CI) of each ocular vessel was calculated by dividing the ocular blood flow by the MAP.

\section{Data analysis}

Data were expressed as mean \pm SE values. The effects of time and blood vessels were examined by repeatedmeasures ANOVA. When a significant $F$ value was detected, this was analyzed further against the baseline value using Scheffé's post hoc test. The level of statistical significance was set at $P<0.05$. All of the statistical analyses were performed using SAS (version 8.2, SAS Institute, Cary, NC, USA) at the Computing and Communications Center, Kyushu University, Japan.

\section{Results}

During passive heat stress, HR, fSBF and external ear temperature increased significantly from the normothermia levels, while MAP and $\mathrm{PaCO}_{2}$ remained unchanged (Table 1).

STRA and SNRA blood flow and RCV blood flow velocity decreased significantly from the normothermia level after $90 \mathrm{~min}$ of passive heat stress in all subjects (Figure 1). Only the RCV blood velocity decreased significantly throughout the heat stress condition. While the blood velocity in the RCV tend to be lower than that in the SNRA at 60 min of passive heat stress $(P=0.06)$, 
Table 1 Parameters during normothermia and passive heat stress

\begin{tabular}{lcccc}
\hline Parameter & Normothermia ${ }^{\mathbf{a}}$ & \multicolumn{3}{c}{ Passive heat stress $^{\mathbf{a}}$} \\
\cline { 3 - 5 } & & $\mathbf{3 0}$ min & $\mathbf{6 0}$ min & $\mathbf{9 0}$ min \\
\hline Heart rate $(\mathrm{bpm})$ & $69.14 \pm 2.29$ & $76.56 \pm 3.01^{*}$ & $83.27 \pm 3.61^{*}$ & $89.39 \pm 4.36^{*}$ \\
Finger skin blood flow $(\%)$ & - & $49.53 \pm 8.83^{*}$ & $66.30 \pm 12.98^{*}$ & $52.39 \pm 11.16^{*}$ \\
Arterial partial pressure of $\mathrm{CO}_{2}(\mathrm{mmHg})$ & $36.40 \pm 0.74$ & $35.73 \pm 0.91$ & $35.07 \pm 0.97$ & $35.55 \pm 0.83$ \\
Mean arterial pressure $(\mathrm{mmHg})$ & $78.06 \pm 2.52$ & $76.40 \pm 2.88$ & $75.52 \pm 2.61$ & $77.07 \pm 3.02$ \\
External ear temperature $\left({ }^{\circ} \mathrm{C}\right)$ & $36.09 \pm 0.19$ & $36.12 \pm 0.19$ & $36.34 \pm 0.19^{*}$ & $36.48 \pm 0.19^{*}$ \\
\hline
\end{tabular}

${ }^{\mathrm{a}}$ Values are means $\pm \mathrm{SE}$.

${ }^{*} P<0.05$ vs. normothermia.

a significant difference was not observed among ocular vessels through the passive heat stress trial. The CI responses for all of the ocular vessels to the heat stress were similar to those of ocular blood flow (Figure 1).

\section{Discussion}

The main finding of the present study was that passive heat stress reduced the blood flow and $\mathrm{CI}$ in the retinal and choroidal vessels, even though the $\mathrm{PaCO}_{2}$ remained unchanged. These results indicate that factors other than $\mathrm{PaCO}_{2}$ influence the ocular circulation in both the SNRA and STRA during passive heat stress.

Decreased ocular blood flow during passive heat stress can be attributed to a sympathetic nervous response.
The cerebral blood flow, which is also sensitive to $\mathrm{CO}_{2}$, did not return to the baseline value on restoration of $P_{\mathrm{ET}} \mathrm{CO}_{2}$ in heat-stress conditions, suggesting that other factors, such as sympathetic nerve activity, may be sustaining the reduction in cerebral blood flow [10]. Sympathetic nerve activity is enhanced by heat stress [11]. The sympathetic nerve solely innervates the choroidal vessels in the ocular circulation. In turn, the ophthalmic artery, which is upstream of the choroidal and retinal vessels, has a rich sympathetic innervation $[2,12]$. Enhanced sympathetic activity may induce vasoconstriction in the ophthalmic artery, consequently decreasing the conductance of both ocular vessels during passive heat stress. This possibility is partly supported by the observed

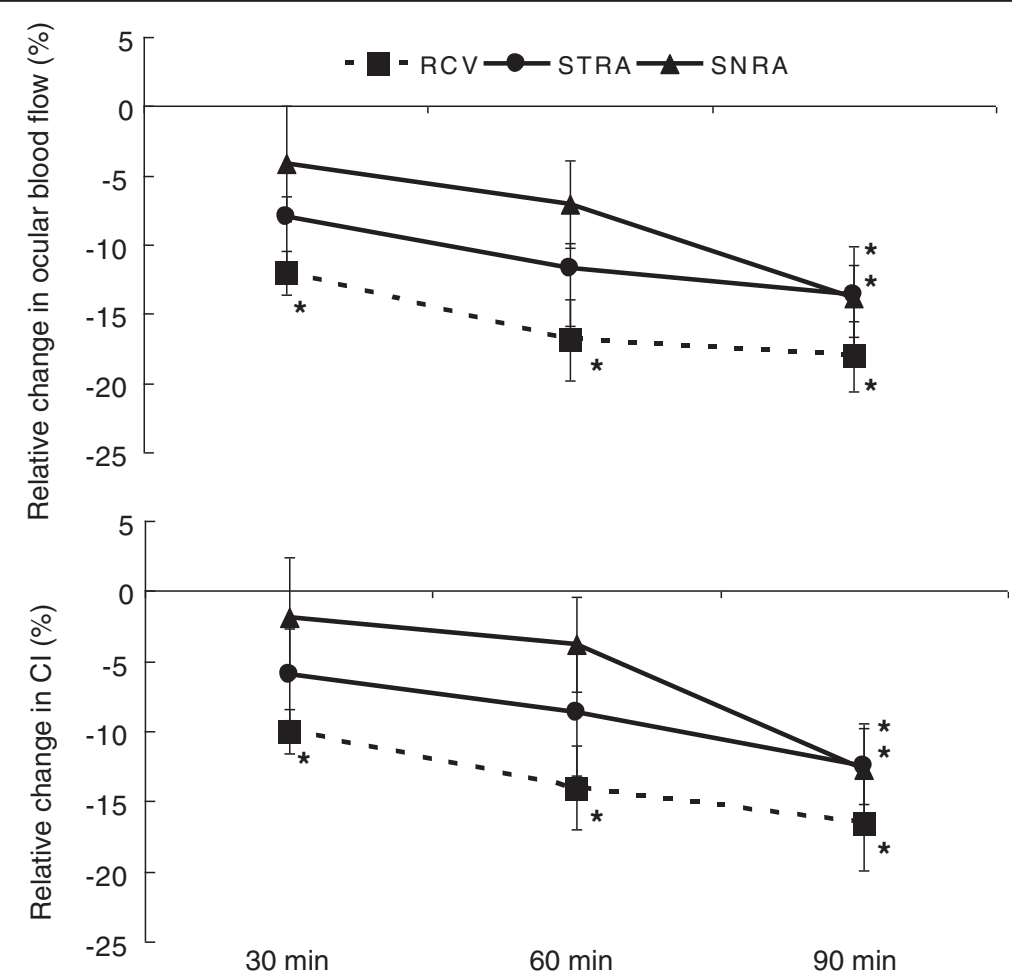

Figure 1 Relative changes in ocular blood flow (upper panel) and conductance index (lower panel) during passive heat stress. Values are means $\pm \mathrm{SE}$. Cl, conductance index; RCV, retinal and choroidal vasculature; STRA, superior temporal retinal artery; SNRA, superior nasal retinal artery. ${ }^{*} P<0.05$ vs. normothermia. 
decrease in fSBF, which should be due to sympathetic activation.

Another factor that can cause a decrease in ocular blood flow is an alteration in the distribution of cardiac output $(\dot{\boldsymbol{Q}})$. $\dot{\boldsymbol{Q}}$ dynamics influence the cerebral blood flow, independently of $\mathrm{PaCO}_{2}$ or cerebral perfusion pressure [13]. The ocular blood flow may also be affected by changes in $\dot{Q}$. More blood is distributed to the skin to aid heat dissipation during hyperthermia [14], which can result in a reduction in the distribution of blood to the ocular circulation during heat stress.

The choroidal circulation responds earlier to heat stress than the retinal circulation. This could be explained by autonomic innervation: as mentioned above, the autonomic innervation is richer for the choroidal circulation than for the retinal circulation [2]. Thus, it is likely that the sympathetic activation induced by heat stress influences the autonomic-rich choroidal circulation earlier than the retinal circulation.

In conclusion, the present results suggest that the blood flow in the retinal and choroidal vasculature is decreased by passive heat stress even without a change in $\mathrm{PaCO}_{2}$. These responses can be attributable to a heatstress-induced enhancement of the sympathetic nerve activity and/or a redistribution of blood. The finding that the choroidal circulation was more responsive than the retinal circulation to heat stress could be explained by differences in sympathetic innervation between these two parts of the ocular circulation.

\section{Abbreviations}

$\mathrm{Cl}$ : Conductance index; fSBF: Finger skin blood flow; HR: Heart rate; LSFG: Laser-speckle flowgraphy; MAP: Mean arterial pressure; $\mathrm{PaCO}_{2}$ : Arterial partial pressure of carbon dioxide; $P_{E T} \mathrm{CO}_{2}$ : End-tidal carbon dioxide; $\dot{Q}$ : Cardiac output; RCV: Retinal and choroidal vasculature; SNRA: Superior nasal retinal artery; STRA: Superior temporal retinal artery; $V_{T}$ : Tidal volume.

\section{Competing interests}

The authors declare that they have no competing interests.

\section{Authors' contributions}

$\mathrm{AM}, \mathrm{HK}$ and $\mathrm{YY}$ contributed to the data collection and data interpretation. $\mathrm{TI}$ and $\mathrm{NH}$ contributed equally to the study design, data collection, data analysis, data interpretation and writing of the manuscript. All authors read and approved the final manuscript.

\section{Acknowledgement}

This study was partly supported by a grant-in-aid for a JSPS Research Fellow (no. 24 7022, to TI).

\section{Author details}

${ }^{1}$ Graduate School of Human-Environment Studies, Kyushu University, Kasuga-koen 6-1, Kasuga, Fukuoka 816-8580, Japan. ${ }^{2}$ Graduate School of Decision Science and Technology, Tokyo Institute of Technology, Ookayama 2-12-1-W9-1, Meguro, Tokyo 152-8552, Japan.

Received: 4 September 2013 Accepted: 19 November 2013

Published: 6 December 2013
References

1. Netter FH: Atlas of Human Anatomy. 4th edition. Philadelphia: Saunders; 2006.

2. Delaey C, Van de Voorde J: Regulatory mechanisms in the retinal and choroidal circulation. Ophthalmic Res 2000, 32(6):249-256.

3. Hayashi $\mathrm{N}$, Ikemura $\mathrm{T}$, Someya $\mathrm{N}$ : Changes in ocular flow induced by hypo- and hypercapnia relate to static visual acuity in humans. Eye Rep 2011, 1(1):e8.

4. Paris G, Sponsel WE, Sandoval SS, Elliott WR, Trigo Y, Sanford DK, Harison JM: Sildenafil increases ocular perfusion. Int Ophthalmol 2001, 23(4-6):355-358.

5. Rowell LB, Brengelmann GL, Murray JA: Cardiovascular responses to sustained high skin temperature in resting man. J Appl Physiol 1969, 27(5):673-680

6. Ikemura $\mathrm{T}$, Hayashi $\mathrm{N}$ : Ocular circulatory response to exhaustive exercise in humans. FASB J 2011, 25:1054.3.

7. Jones NL, Robertson DG, Kane JW: Difference between end-tidal and arterial $\mathrm{PCO}_{2}$ in exercise. J Appl Physiol 1979, 47(5):954-960.

8. Ikemura T, Someya N, Hayashi N: Autoregulation in the ocular and cerebral arteries during the cold pressor test and handgrip exercise. Eur J Appl Physiol 2012, 112(2):641-646.

9. Alm A, Bill A: Ocular and optic nerve blood flow at normal and increased intraocular pressures in monkeys (Macaca irus): a study with radioactively labelled microspheres including flow determinations in brain and some other tissues. Exp Eye Res 1973, 15(1):15-29.

10. Brothers RM, Wingo JE, Hubing KA, Crandall CG: The effects of reduced end-tidal carbon dioxide tension on cerebral blood flow during heat stress. J Physiol 2009, 587(15):3921-3927.

11. Keller DM, Cui J, Davis SL, Low DA, Crandall CG: Heat stress enhances arterial baroreflex control of muscle sympathetic nerve activity via increased sensitivity of burst gating, not burst area, in humans. J Physiol 2006, 573(2):445-451.

12. Bill A: Blood circulation and fluid dynamics in the eye. Physiol Rev 1975, 55(3):383-417.

13. Ogoh S, Ainslie PN: Cerebral blood flow during exercise: mechanisms of regulation. J Appl Physiol 2009, 107:1370-1380.

14. Roddie IC, Shepherd JT, Whelan RF: Evidence from venous oxygen saturation measurements that the increase in forearm blood flow during body heating is confined to the skin. J Physiol 1956, 134(2):444-450.

doi:10.1186/1880-6805-32-23

Cite this article as: Ikemura et al:: Ocular blood flow decreases during passive heat stress in resting humans. Journal of Physiological

Anthropology 2013 32:23.

\section{Submit your next manuscript to BioMed Central and take full advantage of:}

- Convenient online submission

- Thorough peer review

- No space constraints or color figure charges

- Immediate publication on acceptance

- Inclusion in PubMed, CAS, Scopus and Google Scholar

- Research which is freely available for redistribution 